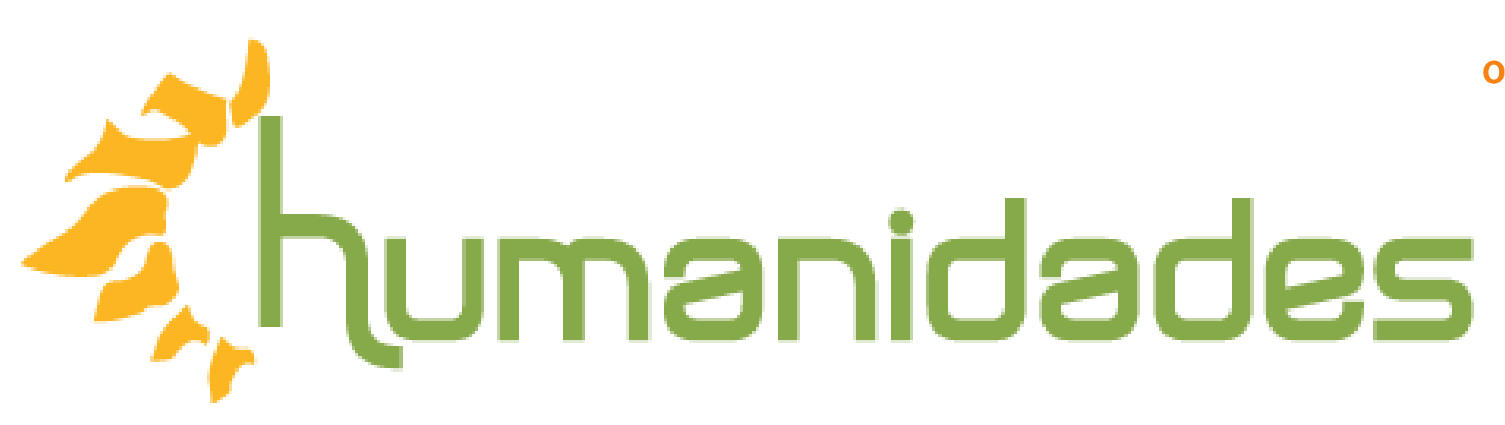

Revista de la Escuela de Estudios Generales, Universidad de Costa Rica

Enero-junio, 2018 •Volumen 8, número 1 • EISSN 2215-3934 •pp. 1-10

Recibido: 08-Noviembre-2017 Aceptado:13-Diciembre-2017

\title{
Brian Connaughton (Coord.) Repensando Guatemala en la época de Rafael Carrera. El país, el hombre y las coordenadas de su tiempo
}

DOI: http://dx.doi.org/10.15517/h.v8i1.31472

\section{Jorge Silva Riquer}

Universidad Michoacana de San Nicolás de Hidalgo

Correo electrónico: jsriquer@gmail.com

Todos los derechos reservados. Universidad de Costa Rica. Esta revista se encuentra licenciada con Creative Commons. Reconocimiento-NoComercial-SinObraDerivada 3.0 Costa Rica. Correo electrónico: humanidades@ucr.ac.cr / Sitio web: http://revistas.ucr.ac.cr/index.php/ humanidades 


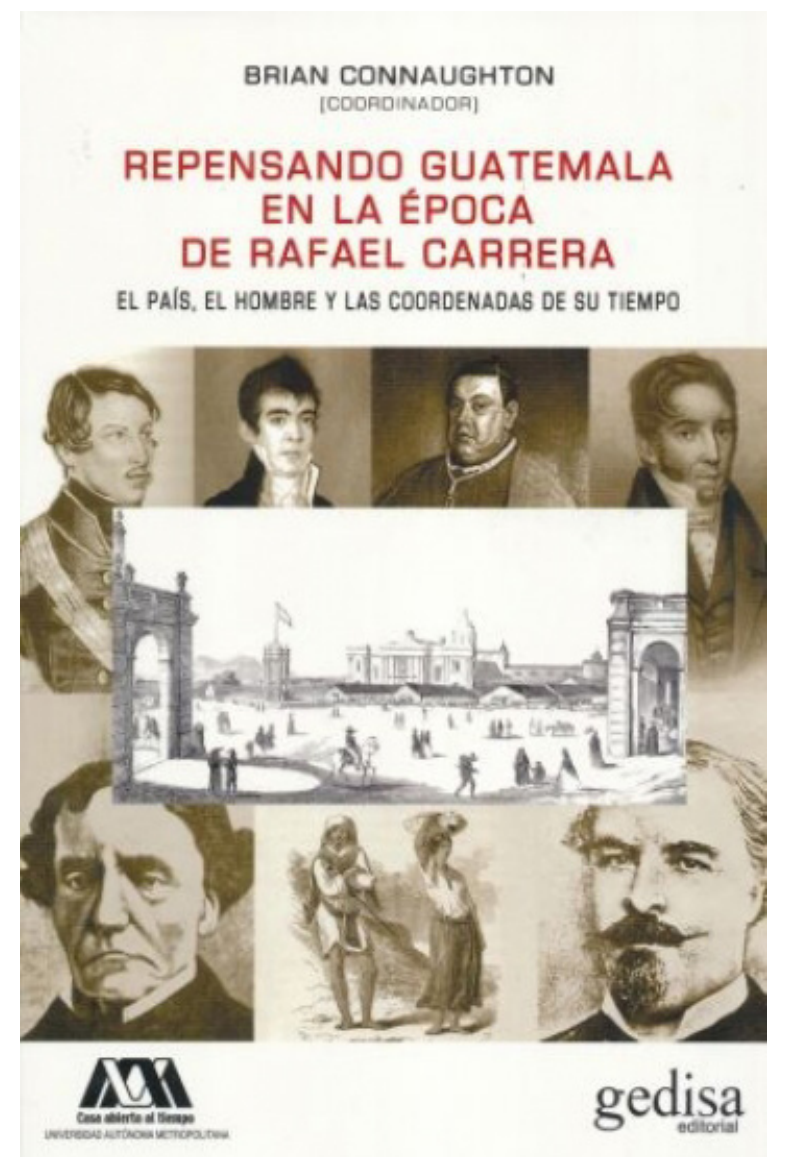

Brian Connaughton (Coord.) Repensando Guatemala en la época de Rafael Carrera. El país, el hombre y las coordenadas de su tiempo Universidad Autónoma Metropolitana I - Gedisa Editorial (Biblioteca Ibroamricana de Pensamiento), 2015

ISBN: 978-84-9784-986-9 
La formación del estado en Guatemala a través del periodo conocido como conservador es abordado por los autores de este libro, son interesantes por varias razones, algunas son: empezar a entender al personaje como parte de un todo histórico; explicar las redes de poder establecidas para la permanencia y establecimiento de un proyecto político-económico; la importancia que tienen para entender esa historia, poco tratada en la historiografía mexicana, pero necesaria por lo cercano a la nuestra y hacer una reflexión comparativa, las similitudes y diferencias son abundantes y es necesario empezar a revisarlas, para conocer mejor la historia de América Central, que sin duda nos atañe. Nos permite, entonces, hacer un ejercicio de deliberación y comparación para establecer puntos de convergencia, discusión y de entendimiento del proceso de construcción del Estado liberal en América Latina. Labor interesante que inició con un Seminario, continuó en un Coloquio y concluyó con la conjunción de los trabajos, una vez discutidos y revisados, por los diversos autores, a través de la coordinación de Brian Connaughton.

Una vez que se adentra en la lectura del texto, la reflexión nos permite entender sobre varios asuntos, uno de ellos es la importancia que tiene haber realizado este estudio del periodo de Rafael Carrera como presidente de Guatemala, máxime, cuando se han realizado varios y, como se indica en la introducción, todos ellos significativos y, con aportes historiográficos, una respuesta es presentada por el mismo coordinador, analizar el proceso desde perspectivas novedosas y poco abordadas, que les permitirán tener otros acercamientos y nuevas interpretaciones sobre este controvertido personaje, o más bien, para decirlo en mejores términos, empezar a definir mejor el proceso histórico, a partir de otras variables sustantivas, no abordadas antes, buscando que los resultados permitan explicarlo en ambos sentido, como un todo, desde la complejidad histórica, una propuesta fundamental del texto y un aporte novedoso.

Entonces desde la introducción nos indican por dónde va el texto y los temas que aborda, lo que nos permite tener una idea clara y precisa, otro aporte es que el coordinador no ceja en su interés de indicarnos y llevarnos por este camino, sino que nos permite conocer la bibliografía sobre el periodo y sus aportes, así, como las necesidades que se abren a partir de ello, en la Introducción y la Coda, ambas, se combinan para darle sentido y explicación al libro. Con ello podemos tener un acercamiento al espacio y tiempo del personaje y de la complejidad del proceso histórico. 
Para ello, me permitiré, hacer una reflexión a partir de los problemas planteados, aunque altere el orden de la presentación.

Los trabajos que conforman este texto abordan una problemática en conjunto, la construcción del estado liberal, no solo a través de la personalidad del personaje, presente en la historia de los siglos XIX y XX, sino las formas que lograron conjuntar, bajo su personalidad, para cubrir varios aspectos sustanciales en el proceso, que les permitieron mantenerse en el poder por varios años y continuar con la construcción estatal, tal fue al caso de Rafael Carrera. Toda una personalidad, con cualidades y habilidades para ejercer el gobierno, militar y civil, por medio de acuerdos con los diversos grupos de poder, que le consintieron permanecer y llevar a cabo el proyecto acordado, son algunos resultados que nos ofrecen.

Es indispensable entender al personaje y su entorno temporal, nos señalan en los textos, pero no entendido como la base fundamental de la permanencia, sino como una pieza más en la compleja red de poder y, ahí está otro de los aportes sustantivos de los autores, donde no solo se menciona al dictador como la pieza fundamental, sino que incluyen y abren el indispensable análisis del contexto histórico guatemalteco, por lo que su lectura se hace más completa de esa realidad histórica.

Sin ser una revisión del proceso "llamado conservador" en Guatemala, los autores abordan desde distintas ópticas y problemas del periodo para darle una explicación más amplia, compleja y convincente, no solo rescatar la personalidad, los actos y la capacidad de un personaje, sino las tramas y negociaciones realizadas entre los diversos grupos participantes que le permitieron llevar a cabo el proyecto de gobierno. Lo que adquiere una dimensión amplia y cercana a otros problemas no tratados hasta ahora, según entiendo de la introducción.

El abordar aspectos económicos, sociales y culturales del periodo les permite tener una interpretación más cabal del mismo, en ese sentido los trabajos que emprenden las diversas problemáticas abren el panorama y nos permiten entender a Carrera como un personaje inserto en una serie de condiciones impuestas, negociadas y propias que le dan una presencia más social y nos permiten entonces realizar comparaciones en torno a la formación del estado liberal del siglo XIX en América Latina. 
Además de evitar extrapolaciones endebles a partir de la personalidad de los "dictadores" que campearon durante ese tiempo y ese espacio, las similitudes podrían ser parciales y podríamos señalar la casi semejanza a partir de esa presencia. Sin embargo, el trabajo que presentamos tiene la cualidad de analizar varios aspectos sustantivos de la formación del estado liberal decimonónico, que de alguna manera habíamos olvidado, o bien, no hemos buscado integrarlos en un todo que nos permita conocer y analizar mejor el proceso, y no el personaje.

Los ejemplos que se presentan en este libro aportan elementos para entender a Carrera y los diversos grupos que lo apoyaron, así, los artículos nos hablan e introducen en las finanzas públicas y de algunos de los sectores económicos existentes, nos permiten acercarnos a una realidad que respondió a los intentos de modernización y que los enfrentó, su manera no la podemos clasificar de "conservadora" bajo el significado que se le ha proporcionado en la historiografía positivista, sino tenemos que entender la resistencia como un proceso social que ante la alteración y los cambios, los actores agrupados en sus identidades asumieron las respuestas conocidas y necesarias de acuerdo a sus necesidades y condiciones.

A que me refiero, los juicios que hemos hecho del retraso, o del poco avance en términos de la construcción liberal, la valoramos de acuerdo a una percepción equivocada del tiempo, o más bien del proceso histórico, esperamos que estos se den de manera consistente y eficientemente. Por ejemplo, los estudios que hablan de las finanzas públicas nos demuestran que estás se mantuvieron ante la imposibilidad de llevar a la práctica los cambios necesarios, las condiciones fueron adversas por muchos factores, que estos autores señalan. Un primer paso fue darle un orden al desorden generado por los intentos de cambio promovidos por los gobiernos anteriores; sin establecer estas medidas, no podría sostenerse ninguna forma de gobierno, el resultado fue un orden y mantenimiento de la estructura fiscal precapitalista, donde los recursos del Estado provenían de los impuestos al comercio y al aguardiente, además de los préstamos y capitaciones de guerra. El resultado nos dice Ornelas, fue la existencia de finanzas sanas incluso el pago de préstamos y la liquidación de la deuda, todo un éxito de ese gobierno y por ende su permanencia.

Los trabajos de Sarazúa y González, nos presentan estudios particulares respecto a la fiscalidad y la participación militar, él primero, y sobre un impuesto y su impacto, él otro, ambos nos arrojan resultados estimulantes en torno a las formas 
que asumieron los diversos grupos y las negociaciones que se llevaron a cabo para resolver la imperiosa necesidad de llevar la calma al territorio después de la Rebelión de la Montaña, así como resolver la organización y funcionamiento de uno de los ingresos consistentes de la hacienda pública, el impuesto a la producción, venta y consumo del aguardiente, sin olvidar los cambios que se llevaron sobre estos para hacerlos más eficaces. Los resultados de estos dos trabajos anuncian las formas de negociación que empezaron a establecerse en estos espacios para mantener el control y obtener ganancias mutuas, o sea, entre el gobierno, al recaudar los impuestos, y los grupos económicos, vía el acceso a la tierra, a los negocios y sobre todo al mantener el monopolio del aguardiente en el territorio.

Otro problema abordado en el libro, es el referido a la relación que se estableció entre el gobierno y sus gobernados con algunas formas de organización y participación, la cual se estableció en ambas direcciones, así los trabajos que abordan la problemática de la vida cotidiana en sus diversas prácticas nos acercan a las posibles respuestas sociales ante las medidas de orden político, económico y social impuestas por la autoridad, pero también a sus formas de respuesta y participación. Con sus trabajos Sagastume y González Galeoti nos ponen en contexto de estos asuntos vitales del periodo, la necesidad de establecer la instrucción y la incorporación de los habitantes al trabajo, que denominan como "costumbres" y, por otro lado, las formas de reorganización de las comunidades y/o pueblos de indios ante los cambios liberales, precisamente después de la Rebelión de la Montaña.

En estos podemos observar las formas que asumieron, ambos grupos, para lograr primero una estabilidad y después establecer los principios que en ese momento se aceptaban como los ideales para darle el impulso a la sociedad en sus diversas esferas. Por un lado, la definición de vagancia, ociosidad y "mal entretenimiento", mantuvo los principios ilustrados, pero con cambios a lo largo del periodo, para ir estableciendo los principios utilitaristas propios de ese liberalismo inicial. De ahí, que la discusión de Sagastume, nos permita vislumbrar la dicotomía entre la participación de los juegos y la constante del trabajo como parte de las formas que se aplicaron. Mientras que el capítulo sobre la comunidad de Santa Catarina, nos presenta una forma de solución al conflicto agrario, el eterno problema de la tenencia de la tierra, las formas que se asumieron permitieron por lo menos, nos dice el autor, lograr una pacificación y forma de recreación de las prácticas indígenas, una propuesta interesante ya que se restablecieron las formas cotidianas de dicha comunidad. 
En estos dos trabajos se logra ponernos en contexto de las formas políticas y sociales que aplicó el gobierno de Carrera para lograr los objetivos planteados, si bien, no fueron similares, si buscaron solucionar dos asuntos de primera necesidad, uno referido al orden y la necesidad de establecer un mercado de trabajo, bajo los principios utilitaristas de la época y, el otro, resolver de manera eficiente el problema bélico de una de las comunidades de los Altos como parte fundamental de la pacificación de ese territorio para establecer la estabilidad política que le permitió a Carrera gobernar por varias décadas.

Otro texto es el que aborda el problema de las mujeres, el que tiene la cualidad de ofrecernos una relación rica en descripciones, por ejemplo: como las mujeres asumieron el periodo y los diversos avatares a los que se vieron enfrentadas, la narración es rica en el rescate de los textos de viajeros y demás. La autora hace un intento por introducir la propuesta de Bandidos presentada por Hobsbawm, interesante, pero que, deberá seguir elaborando para darle una conformación más sólida y consistente; el llevar a cabo el acercamiento entre la propuesta de bandido social y, las evidencias, demuestran un objetivo que la autora se ha planteado y que no acaba de resolver, por lo que esperamos, que nos entregue en futuras publicaciones, resultados más contundentes, mientras el acercamiento es interesante y plantea varios aspectos a investigar.

La historia política está presente de manera interesante, pues a partir de revisar y analizar la transformación que se dio en el personaje de Carrera y de la participación del grupo denominado "los pardos" como parte integrante de su contingente, se abren otras vertientes de investigación, que arrojan nuevas interpretaciones sobre el proceso de organización y participación de ese personaje y los diversos actores que tuvieron una incidencia. Así, el trabajo de Connaughton hace un análisis minucioso de la transformación del caudillo, lo llama el paso del "indio a presidente vitalicio" y, nos adelanta desde el título de su texto, la utilización hermenéutica de la llamada cultura política.

Un proceso que revisa, estudia y analiza a partir de varias evidencias de viajeros y demás, sin perder de vista las formas necesarias de la organización que llevó a cabo para establecer el estado liberal "conservador". Así nos presenta la metamorfosis política de un personaje rodeado de otros actores, que le permitieron entender las necesidades a las que se enfrentaba y resolverlas, de manera acordada y establecer un gobierno congruente a los planteamientos y conocimientos de los participantes.

Esa transformación política de Carrera estuvo relacionada con los grupos y personajes que 
encontraron en su persona las formas de organizar una propuesta de gobierno acorde a las necesidades e interés particulares, dentro de ellos varios grupos fueron los beneficiarios. Por otro lado, nos presenta al caudillo como un ser capaz de entender y asumir la práctica política como parte indispensable de un proyecto, que fue construyendo conforme avanzó el siglo, desde la justicia, el fisco, el gobierno y la seguridad, como ha quedado demostrado en los capítulos que conforman el libro.

Los dos primeros trabajos que inician el libro, hacen una revisión sobre el proceso de la formación del estado liberal en Guatemala, su intención es darnos una visión general, donde la narración de los hechos, es la forma de presentarnos el escenario donde "el caudillo" se desenvolvió y logró establecer un gobierno por varios años; donde la condición estuvo marcada por su personalidad en los logros y retrocesos registrados. La propuesta de estos trabajos, se ubican en entender esa continuidad desde el periodo colonial, hasta el periodo "conservador", con la presencia intermedia de un gobierno liberal federal, donde la Capitanía General de Guatemala tenía una condición menor que los virreinatos americanos, por lo que la reforma del siglo XVIII tuvo una presencia distinta, provocando los resultados expuestos en estos textos.

Lo significativo fue la continuidad en torno a varias de las prácticas políticas establecidas por la Corona española, con los intentos de cambio iniciados por el gobierno criollo liberal federal, con tintes integracionistas, ya fuera a México, o, a Centroamérica, los resultados de ese primer intento al parecer "fueron brutales", según nos narran en su investigación.

La posibilidad de la formación de un gobierno liberal desembocó en un conflicto entre los diversos grupos, donde el establecimiento de un gobierno estable y legítimo mantuviera la integración territorial y social, en ese sentido podemos decir que la reforma del siglo XVIII no cumplió con su cometido, el problema se agudizó con ese primer gobierno liberal y desembocó en una inconformidad que dio paso a la llamada revuelta de "Los Altos", principalmente de la población de Mita, entre otros.

Ese proceso permitió la consolidación de familias que, a través de esta respuesta de inconformidad a las medidas del gobierno de Gálvez, permitieron que se hicie 
Brian Connaughton (Coord.) Repensando Guatemala....

ran presentes en varias prácticas desde las militares, hasta las económicas, , así lo presenta Jefferson en su trabajo, la familia Mexia, estuvo presente en la organización y formas de participación contrarias a las medidas y aplicación del proyecto liberal federal, impulsando la propuesta de integración ya mencionada. El trabajo hace un acercamiento a la interpretación que se tenía de lo que debería ser América y cómo reordenarla, sin duda, los grupos de "pardos", "mulatos" e indios tuvieron una presencia significativa en la respuesta armada iniciada en 1838 en contra del presidente y la instauración del caudillo Carrera, como parte de un proyecto más "americano", aunque la autora no lo haga explícito.

El texto tiene varias aportes y cualidades que lo hace un trabajo de consulta para conocer y sobretodo, empezar a entender la formación del estado liberal en Guatemala, nos presentan a un caudillo de "carne y hueso", donde los intereses, las incapacidades y las propuestas están presentes en la construcción de un proyecto, lo que nos permite entonces reconocer la condición de actores históricos, por su ubicación en el tiempo, bajo los avatares constantes de la necesidad urgente de reorganizar un estado, con su gobierno, sus finanzas públicas, su justicia y seguridad, condiciones que son sustanciales para la permanencia en el poder.

Ya la filosofía política lo había empezado a discutir y a plantear desde hacía algunos años antes, y en este trabajo están presentes, como una condición básica para realizar la investigación, otra cualidad importante; la integración, aunque fuera incipiente y tenue, nos permite tender argumentos que nos acercan a una problemática que va más allá de la historiografía positivista del "líder preclaro y poderoso", para presentarnos a un caudillo inserto en su tiempo y sus condiciones, pero con la habilidad y conocimiento de la cultura política que le permitió plantear e intentar resolver los apremios más urgentes y lograr establecer un gobierno más estable, en la conjunción de otros actores políticos, no podía haber sido de otra manera. La discusión si fue conservador o no, adquiere una dimensión más analítica y menos maniquea, felicidades por ese logro.

Por todo lo anterior recomiendo ampliamente la lectura y consulta del texto, felicito a los autores y al coordinador por el trabajo realizado, puedo decir a partir de la lectura he aprendido a entender el proceso de formación del gobierno liberal guatemalteco en el siglo XIX, pero, sobre todo me han despertado inquietudes y preguntas, lo que hace más valioso el trabajo en sí. 


\section{¿Cómo citar este artículo?}

Silva Riquer, J. (Enero-junio, 2018). Brian Connaughton (Coord.) Repensando Guatemala en la época de Rafael Carrera. El país, el hombre y las coordenadas de su tiempo . Revista humanidades, 8(1), 1-10 doi: http:// dx.doi. org/10.15517/h.v8i1.31472 